\title{
CDISC Glossary Terminology
}

National Cancer Institute

\section{Source}

National Cancer Institute. CDISC Glossary Terminology. NCI Thesaurus. Code C67497.

The terminology that includes terms relevant to the Clinical Data Interchange Standards Consortium Glossary group. 